\title{
EVALUACIÓN DE Beauveria bassiana PARA EL COMBATE DE INSECTOS PLAGA EN MAÍZ ALMACENADO1
}

\author{
Juan Diego Molina 2, José Raúl Espinal ${ }^{2}$
}

\begin{abstract}
RESUMEN
Evaluación de Beauveria bassiana para el combate de insectos plaga en maíz almacenado. El objetivo de este estudio fue evaluar la patogenicidad de aislamientos de Beauveria bassiana, para controlar los insectos plaga Prostephanus truncatus y Stophilus zeamais, en mazorcas de maíz almacenado. Se evaluó también la patogenicidad del hongo sobre Teritriosoma nigrescens, depredador natural de P. truncatus. Se mezclaron conidios secos del hongo con kaolinita y se aplicaron a trojas de maíz de 450 mazorcas acomodadas en capas de 50. Se montaron dos ensayos, en el primero se evaluaron aplicaciones del hongo al momento de almacenar el maíz, y en el segundo se evaluaron aplicaciones del hongo realizadas después de almacenar el maíz. La mortalidad de $P$. truncatus fue cuatro veces mayor en el ensayo donde se aplicó el hongo en forma preventiva. La mortalidad de $S$. zeamais fue el doble en el tratamiento donde se aplicó el hongo al momento de inocular con $P$. truncatus. Los niveles de mortalidad por B. bassiana fueron mayores en P. truncatus que en $S$. zeamais, pero $S$. zeamais, pero este último mostró un $20 \%$ más de infección que el primero.
\end{abstract}

\begin{abstract}
Evaluation of Beauveria bassiana for the control of insect pest in stored maize. The objective of this study was to evaluate the pathogeneicity of some isolates of Beauveria bassiana for the control of insect pests such as Prostephanus truncatus and Stophilus zeamais, in stored maize cobs. Beauveria pathogenecity was also evaluated on Teritriosoma nigrescens, a natural predator of $P$. truncatus. Conidia of the fungi were mixed with kaolinite and applied to stored maize, 450 cobs arranged in layers of 50. Two trials were established, in the first trial applications of the fungi was done at the moment of storing the maize. In the second trial, applications of the fungi were evaluated after storing the maize. The mortality of $P$. truncatus was four times higher in the trial where the fungi was applied as a preventive treatment. The mortality of $S$. zeamais was doubled in the treatment where the fungi was applied at the moment of inoculating with $P$. truncatus. Mortality levels caused by $B$. bassiana were higher in P. truncatus than in S. zeamais, but S. zeamais showed an infection $20 \%$ larger.
\end{abstract}

\section{INTRODUCCIÓN}

En América Latina y Africa el maíz es uno de los principales cultivos utilizados para la alimentación humana. En estas regiones, se ha encontrado que en los sistemas tradicionales de almacenamiento de grano seco se pueden llegar a tener severas pérdidas debido al mal manejo de los granos. El mal secado y sobre todo, el daño ocasionado por el ataque de insectos en almacenamiento, son las principales causas de las pérdidas.

Las pérdidas en Latinoamérica son causadas por insectos primarios como Sitophilus zeamais Motsh (Coleoptera: Curculionidae) que es la plaga principal asociada con las pérdidas de almacenamiento de maíz en las regiones tropicales del continente (Ríos, 1991). Sin embargo, Prostephanus truncatus (Horn) (Coleoptera: Bostrichidae), el mayor barrenador de los granos, puede causar pérdidas mayores en caso de no tener huéspedes alternos como árboles de los géneros Spondia y Bursera (Novillo, 1991).

Prostephanus truncatus es una plaga ocasional de maíz almacenado tradicionalmente en Mesoamérica, su lugar de origen. Sin embargo, este insecto fue introducido accidentalmente en Africa a principios de la década de los ochenta. Actualmente, se encuentra distribuida en Africa del este y del oeste, convirtiéndose en un problema muy importante en maíz y yuca almacenados (Markham et al., 1991). Investigaciones en Tanzania

\footnotetext{
Trabajo de tesis para optar al título de Ing. Agr. de la Escuela Agrícola Panamericana, El Zamorano.

2 CADECA-COVEPA, Apdo. Postal 1050, Tegucigalpa, Honduras.

3 Citesgran, Agronomía, EAP, Zamorano. Apdo. Postal 93, Tegucigalpa, Honduras.
} 
informan que $P$. truncatus provoca pérdidas máximas del 17,4 por ciento en el peso seco después de seis meses de almacenamiento y de 41,2 por ciento después de ocho meses (Keil, 1988).

El objetivo general de este estudio fue evaluar una táctica de control biológico para $P$. truncatus y $S$. zeamais que no dañan a Teretriosoma nigrescens (Lewis) (Col:Histeridae), la cual sea de bajo riesgo a la salud humana a través de la evaluación de aislamientos de $B$. bassiana a nivel de laboratorio y su aplicación en almacenes tradicionales de maíz infestados con Prostephanus truncatus..

\section{MATERIALES Y MÉTODOS}

\section{Ensayo I. Evaluación de la patogenicidad de cinco aislamientos de Beauveria bassiana contra Prostep- hanus truncatus y Teretriosoma nigrescens.}

Este estudio se realizó en el laboratorio de fitopatología del departamento de protección vegetal (DPV), de la Escuela Agrícola Panamericana (EAP), Zamorano, Honduras. Se realizaron bioensayos con cinco aislamientos del hongo entomopatógeno $B$. bassiana, en dos formulaciones: conidios secos y conidios en emulsión de aceite. Estos aislamientos provenían de plagas de los órdenes Coleóptera y Lepidóptera.

Establecimiento de los bioensayos. Los adultos de $P$. truncatus utilizados en los bioensayos tenían una edad aproximada de 25 a 35 días de emergidos. Para asegurar esto, se colocaron 50 adultos a ovopositar en 100 g de grano de maíz durante 21 días. Luego, se removieron los 50 adultos. Se esperó entre 30 y 35 días para que los adultos de la siguiente generación emergieran. Los nuevos adultos se mantuvieron en la cámara de cría del laboratorio de entomología del CITESGRAN durante un día, bajo las siguientes condiciones controladas: humedad relativa $70 \pm 7 \%$ y temperatura de $27 \pm$ $2^{\circ} \mathrm{C}$. Estas condiciones se verificaron semanalmente por medio de un higrotermógrafo. Se les proporcionó 12 horas de oscuridad y 12 de luz por día (Calix 1995). Los $T$. nigrescens provenían de crías mantenidas en el laboratorio en granos de maíz infestados con $P$. truncatus.
Las pruebas se montaron en una cámara de flujo laminar, en un cuarto aséptico, para evitar que los insectos se infectaran con otros microorganismos. En $P$. truncatus se probaron todos los aislamientos, mientras en $T$. nigrescens sólo se probaron los aislamientos de cosmopolites sordidus y de Mycotech (formulación en aceite)(Cuadro 1), porque éstos causaron una mayor mortalidad en $P$. truncatus. Como testigo para ambas especies, individuos de $P$. truncatus y $T$. nigrescens se sumergieron sólo en agua destilada.

Para cada aislamiento se preparó una solución acuosa de $1 \times 10^{8}$ conidios $/ \mathrm{ml}$ de B. bassiana. Luego, los adultos de $P$. truncatus y $T$. nigrescens (45 individuos por especie para cada aislamiento), se sumergieron durante 30 segundos en la solución. Estos adultos se colocaron individualmente en cajas de plástico en compartimientos separados. Las dimensiones de las cajas fueron: $14 \mathrm{~cm}$ de largo, siete $\mathrm{cm}$ de ancho y dos $\mathrm{cm}$ de alto. Se les colocó un tubo de aluminio de un centímetro de diámetro por 2,2 cm de alto, para evitar que los insectos pudieran subir por las paredes de los compartimientos. Se colocó $0,2 \mathrm{~g}$ de maíz quebrado por compartimiento para la alimentación de los adultos de $P$. truncatus y una larva de $P$. truncatus en el maíz quebrado para la alimentación del depredador T. nigrescens.

Para mantener la humedad relativa se le colocó un pedazo de toalla de papel húmedo por dos días para que el hongo tuviera entre $90 \%$ y $100 \%$ de humedad relativa, condiciones adecuadas para su desarrollo. Luego, cada caja se colocó en la cámara incubadora. Estas condiciones se controlaban con ayuda de un higrotermógrafo a una temperatura de $28 \pm 2^{\circ} \mathrm{C}$ y una humedad relativa de $70 \pm 7 \%$ durante una semana.

\section{Revisión de los bioensayos}

Los insectos se revisaron diariamente por un período de una semana. Los adultos muertos se retiraban de las cajas y se sumergían por un minuto en una solución de hipoclorito de sodio al 0,5\%, para la eliminación de cualquier microorganismo presente en la superficie del insecto. Posteriormente se enjuagaban con agua destilada durante un minuto para eliminar el exceso de desinfectante. Los adultos de $P$. truncatus y $T$. nigrescens se colocaron en platos Petri que contenían un pedazo de toalla de papel húmedo, en la cámara de incubación ba-

Cuadro 1. Tiempo letal medio de los cinco aislamientos de conidios de B. bassiana provenientes de plagas de Coleóptera y Lepidóptera.

\begin{tabular}{lccccc}
\hline & $\begin{array}{c}\text { Hypothenemus } \\
\text { hampeii }\end{array}$ & Anthonomus grandis & Cosmopolites sordidus & $\begin{array}{c}\text { Formulación } \\
\text { en aceite }\end{array}$ & Plutella xylostella \\
\hline TL 50 (hr) & 92,3 & 98,8 & 110,5 & 117 & 124,1 \\
\hline
\end{tabular}


jo condiciones controladas de $70 \pm 7 \%$ humedad relativa y $28 \pm 2^{\circ} \mathrm{C}$ de temperatura. $\mathrm{Al}$ cabo de una semana se observaba si había crecimiento de algún hongo entomopatógeno, el cual era aislado en un medio de cultivo de papa dextrosa agar (PDA) a razón de diez mil por plato Petri, con el antibiótico estreptomicina $(0,05 \mathrm{~g} / \mathrm{l}$ de medio), para permitir la observación e identificación del hongo $B$. bassiana. La identificación se hizo por medio de observaciones visuales del hongo (Loreck, 1995).

En cada prueba se contaron diariamente los insectos muertos, se calcularon los porcentajes de mortalidad diaria y acumulada.

Para $P$. truncatus se determinó el tiempo letal 50 $\left(\mathrm{TL}_{50}\right)$ o sea el tiempo necesario para que muera el $50 \%$ de los adultos $\left(\mathrm{TL}_{50}\right)$ a una concentración del hongo de $1 \times 10^{8}$ conidios por mililitro. Se utilizó el programa Probit Analysis Program para calcular la $\mathrm{TL}_{50}$.

Ensayo II y III. Evaluación de Beauveria bassiana en kaolinita para el control de $P$. truncatus y $S$. zeamais en maíz almacenado por pequeños productores.

\section{Localización del estudio:}

Este estudio se realizó en tres comunidades en el Valle del río Yeguare, departamento de Francisco Morazán, Honduras a una altitud promedio de $800 \mathrm{~m}$; con una temperatura máxima y mínima promedio de 29,4 ${ }^{\circ} \mathrm{C}$ y $19{ }^{\circ} \mathrm{C}$ respectivamente y una precipitación anual de $1100 \mathrm{~mm}$, distribuida entre mayo y noviembre. Las comunidades fueron El Suyatillo, San Francisco y Galeras. Se escogieron por tres razones. Primero, por estar a una distancia promedio de no más de ocho $\mathrm{km}$ de la EAP; lo cual era necesario porque los muestreos se realizaron semanalmente. Segundo, el maíz es producido por pequeños agricultores que utilizan este grano para su alimentación diaria. Tercero, estos agricultores habían colaborado con el Proyecto Prostephanus y presentaban las mismas condiciones en sus hogares que los campesinos que utilizaban sistemas tradicionales de almacenamiento.

\section{Diseño del Estudio}

Se escogió el aislamiento de $B$. bassiana proveniente del picudo del algodón Anthonomus grandis porque fue el que causó la mayor mortalidad sobre $P$. truncatus y la menor mortalidad en $T$. nigrescens. Para montar cada ensayo, se fabricaron trojas de madera de orilla de pino para simular el almacenamiento tradicional. Las dimensiones de las trojas fueron de $94 \mathrm{~cm}$ de largo, $56 \mathrm{~cm}$ de ancho y $57 \mathrm{~cm}$ de alto. El maíz que se utilizó fue de la variedad Honduras Planta Baja (HPB), el cual es cultivado por los agricultores de la zona suroriental de Honduras. En cada troja se colocaron 450 mazorcas en capas de 50 mazorcas cada una arregladas horizontalmente. Se muestreó la capa superior semanalmente por un período de siete semanas. Conidios secos del hongo B. bassiana se mezclaron con kaolinita (arcilla inerte) y se aplicaron a una dosis de $0,125 \mathrm{~kg}$ de la mezcla por capa a una concentración de 1x 1010 conidios por troja.

Se establecieron dos ensayos en octubre de 1996:

a) Ensayo II. Trojas infestadas con $P$. truncatus al momento de la aplicación del producto

El objetivo fue probar si aplicaciones preventivas del hongo (al momento de almacenar el maíz) protegerían a las mazorcas del ataque de $P$. truncatus al llegar estos a las trojas. Las trojas se montaron con mazorcas de maíz fumigadas por diez días con Phostoxin (fosfuro de aluminio) a una dosis de una pastilla por $\mathrm{m}^{3}(57 \%$ de ingrediente activo). Se aplicó la kaolinita con conidios de $B$. bassiana por capa, realizándose la inoculación con P. truncatus al momento de montarse las trojas (200 adultos por capa). Los insectos tenían una edad comprendida entre los 25 y 35 días de emergidos.

Se utilizaron ocho trojas en total de diferentes agricultores para evitar el movimiento de insectos entre los tratamientos. A cinco se les aplicó la kaolinita con conidios del hongo y tres trojas como testigos. El arreglo experimental fue completamente al azar (DCA).

b) Ensayo III: Trojas inoculadas con P. truncatus un mes antes de la aplicación de la kaolinita con conidios del hongo

El objetivo fue probar si aplicaciones curativas del hongo (después de almacenado el maíz) protegerían de un mayor daño las mazorcas al encontrarse $P$. truncatus en el interior de las mismas. Las trojas se montaron con mazorcas sin fumigar, las cuales se inocularon con cuatro adultos de $P$. truncatus por mazorca un mes antes de montarse las trojas.

Se usaron cuatro trojas que se les aplicó la kaolinita con conidios del hongo y cuatro como testigo, teniendo una troja por agricultor (ocho agricultores), en un diseño completamente al azar (DCA).

\section{Recolección y análisis de muestras.}

Al momento de montar los ensayos se tomó una muestra de 20 mazorcas a utilizar en los dos ensayos. Se 
determinó la pérdida de peso, el número de individuos de $P$. truncatus, $S$. zeamais y $T$. nigrescens así como el contenido de humedad de los granos. La capa superior de mazorcas de cada troja, se muestreó una vez por semana. De las 50 mazorcas, 20 escogidas al azar se embolsaron y se llevaron al laboratorio para su análisis. A cada muestra se le realizó un conteo inicial de insectos, que se encontraban fuera de las mazorcas al momento de llegar la muestra al laboratorio. Luego, las mazorcas se destuzaron y se desgranaron. Los granos se pesaron y tamizaron para separar los insectos adultos de P. truncatus y S. zeamais. Se calculó el número de insectos por kilogramo de muestra.

En cada muestra a las cuales se les aplicó la kaolinita con conidios del hongo. Todos los adultos muertos de $P$. truncatus y $S$. zeamais encontrados se aislaron en una cámara húmeda para el cálculo del porcentaje de mortalidad debido al B. bassiana. Lo anterior no se hizo con los tratamientos testigo, debido a que en estudios previos realizados por el proyecto, no se habían encontrado evidencias de algún hongo entomopatógeno en insectos recolectados, en el campo o criados en el laboratorio.

En la última semana de muestreo en ambos ensayos, se tomaron 50 insectos vivos de $P$. truncatus para determinar si el porcentaje de mortalidad por $B$. bassiana era diferente al obtenido por el muestreo de sólo los insectos muertos. Se pusieron los insectos en frascos individuales con maíz quebrado $(0,2 \mathrm{~g})$ y se determinó la mortalidad dos semanas después. Los insectos muertos se colocaron en platos Petri por una semana con un pedazo de toalla de papel húmedo en la cámara de cría, para observar si había esporulación del hongo B. bassiana. De cada muestra de maíz desgranado se obtenían tres submuestras de $250 \mathrm{~g}$ cada una, para medir su contenido de humedad por medio del medidor Motomco Meter Aerglide N 919. El resto de la muestra se utilizó para obtener el porcentaje de pérdida usando el método de conteo y pesado de 1000 granos por medio de la siguiente fórmula:

$\%$ de pérdida de peso $=\frac{(\text { PGS } \times \text { NGD })-(\text { NGS } \times \text { PGD })}{\text { PGS x } 1000}$

PGS $=$ Peso de granos sanos.

NGD= Número de granos dañados .

NGS $=$ Número de granos sanos.

$\mathrm{PGD}=$ Peso de grano dañado.

\section{Análisis estadístico.}

Las variables que se analizaron fueron el número de $P$. truncatus y $S$. zeamais por kilogramo y pérdida de peso. En el Ensayo II se normalizaron las variables de pérdida y densidad de $P$. truncatus por medio del logaritmo natural, y en el Ensayo III las variables pérdida y densidad de $S$. zeamais por logaritmo natural y densidad de $P$. truncatus por raíz cuadrada. Se analizó cada variable usando un modelo de medidas repetidas en el tiempo por medio de un análisis de varianza (ANDEVA) $(\alpha=0,05)$ con ayuda del programa Statistical Analysis System (SAS). Para evaluar si había algún efecto de los tratamientos en la población de las plagas a través del tiempo, se calculó el número de insectos días usando el programa ENSTAT (Pedigo y Zeiss, 1996) para calcular el área debajo de la curva del número de insectos contra la fecha. Posteriormente esta nueva variable se analizó usando un ANDEVA en el programa Statistical Analysis System.

\section{RESULTADOS}

Ensayo I. Patogenicidad de cinco aislamientos de Beauveria bassiana en Prostephanus truncatus y Teretriosoma nigrescens.

Al analizar la patogenicidad de los cinco aislamientos de $B$. bassiana contra $P$. truncatus todos causaron un porcentaje de mortalidad similar. El aislamiento de A. grandis fue el que causó la mayor mortalidad con $86,7 \%$ y en segundo lugar el de Cosmopolites sordidus (picudo del banano) con $84,4 \%$, mientras que en el testigo no hubo mortalidad (Figura 1). Por lo tanto los aislamientos provenientes de A. grandis y C. sordidus fueron los escogidos para ser probados contra T. nigrescens enemigo natural de $P$. truncatus.

También en esta prueba con T. nigrescens, se obtuvieron porcentajes de mortalidad similares con los dos aislamientos. El aislamiento de Anthonomus grandis $(62,2 \%)$ causó el menor porcentaje de mortalidad al depredador T. nigrescens. Con el aislamiento de Cosmopolites sordidus se obtuvo una mortalidad de $66,7 \%$, mientras que en el testigo no hubo mortalidad (Figura 2).

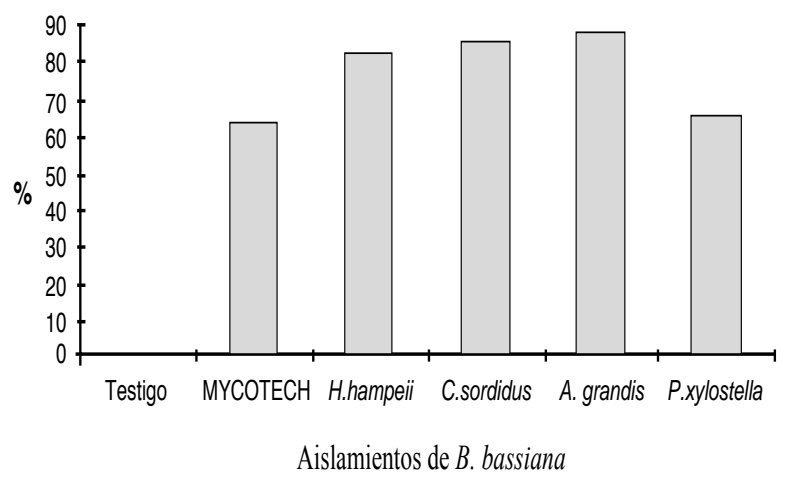

Figura 1. Porcentajes de mortalidad de P. truncatus provocados por los aislamientos de $B$. bassiana. 


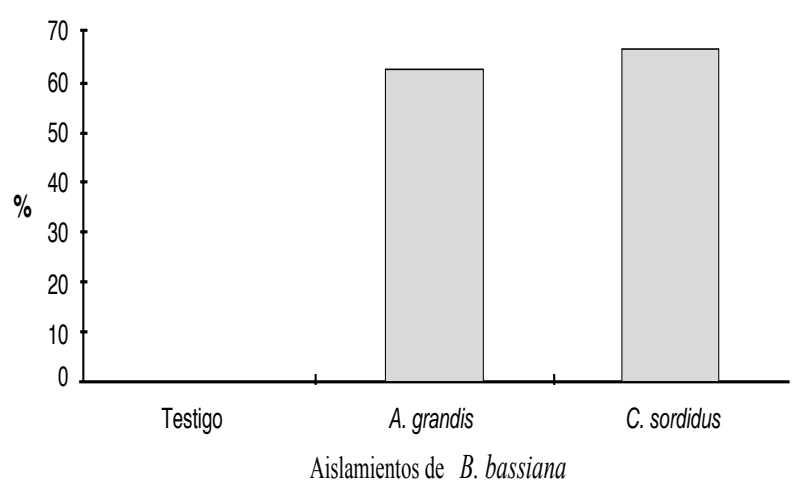

Figura 2. Porcentaje de mortalidad de T. nigrescens debido a los aislamientos de B. bassiana.

Con los resultados obtenidos, se escogió el aislamiento de Anthonomus grandis para ser probado en el campo. Los aislamientos de $H$. hampeii y A. grandis tuvieron las menores TL 50 para $P$. truncatus con 92,3 y 98,8 horas respectivamente, mientras que los aislamientos de C. sordidus, P. xylostella y la formulación en aceite (MYCOTECH) tardaron más de 100 horas en matar a la mitad de la población (Cuadro 1 y Figura 3).

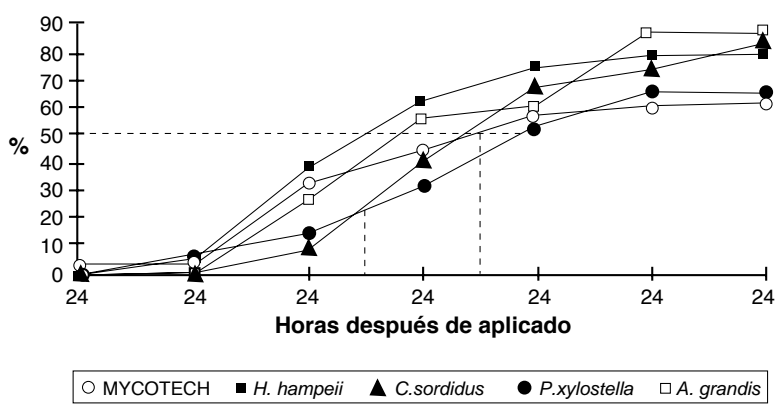

Figura 3. Tiempo letal medio de los cinco aislamientos de B. bassiana en P. truncatus.

Ensayos II y III. Evaluación de Beauveria bassiana en kaolinita para el control de Prostephanus truncatus y Sitophilus zeamais en almacenes tradicionales de maíz.

\section{Ensayo II. Trojas infestadas con Prostephanus trun- catus al momento de la aplicación del producto.}

Las diferencias entre tratamientos y semanas de muestreo no fueron significativamente diferentes a través del tiempo para las variables número de insectos $/ \mathrm{kg}$ (ambas especies) por kilogramo de grano y pérdida de peso $(\alpha=0,05)$. No hubo una interacción significativa entre fecha de muestreo y los tratamientos para ninguna de las variables analizadas.
Las poblaciones de $P$. truncatus no difirieron significativamente entre tratamientos y semanas de muestreo a través del tiempo (Figura 4).

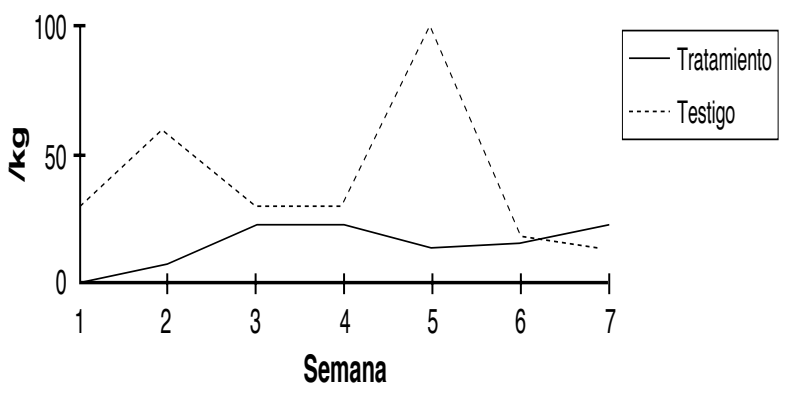

Figura 4. Variación del número de adultos de $P$. truncatus en trojas aplicadas con B. bassiana más kaolinita y trojas testigo en maíz fumigado previa la inoculación con P. truncatus.

Las poblaciones de $S$. zeamais tampoco difirieron significativamente entre tratamientos y semanas de muestreo a través del tiempo (Figura 5).

En las variables días insecto $P$. truncatus, días insecto $S$. zeamais y pérdida de peso acumuladas no se encontraron diferencias estadísticas significativas entre tratamientos $(\alpha=0,05)$.

Aunque la aplicación del producto no afectó significativamente las poblaciones de $P$. truncatus y de $S$. zeamais, sí hubo una infección de individuos de ambas plagas por B. bassiana. Las muestras de insectos muertos indicaron que $B$. bassiana causó una mortalidad promedio de $1,38 \%$ en $P$. truncatus y de $0,6 \%$ en $S$. zeamais en las siete semanas de muestreo. En la muestra viva de $P$. truncatus en el último muestreo, se encontraron similares porcentajes de mortalidad (1,98\%) (Figura 6).

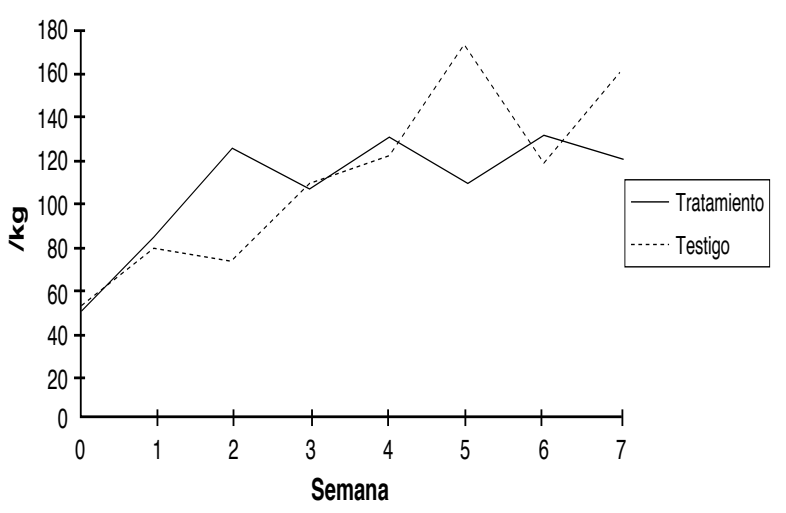

Figura 5. Variación del número de adultos de $S$. zeamais en trojas tratadas con $B$. bassiana más kaolinita y trojas testigo en maíz fumigado previa la inoculación con P. truncatus. 


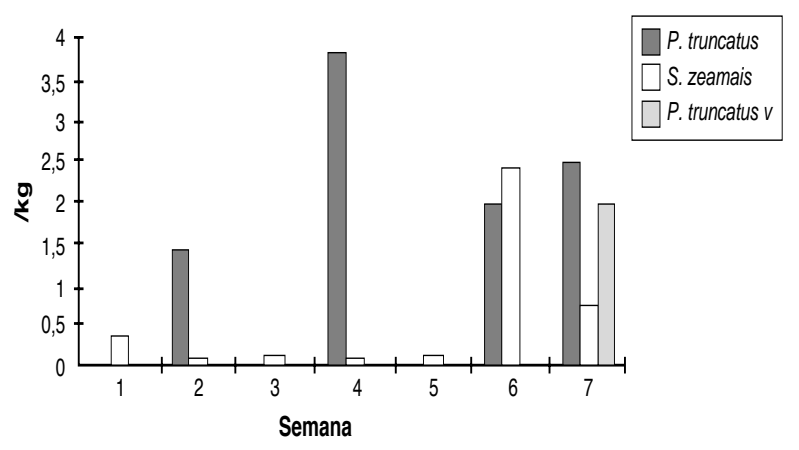

Figura 6 Porcentaje de mortalidad de P. truncatus y $S$. zeamais en trojas aplicadas con $B$. bassiana más kaolinita en maíz fumigado previa inoculación con $P$. truncatus $(P$. truncatus $\mathrm{v}=$ muestra viva de insectos).

Las pérdidas de peso no difirieron significativamente entre tratamientos y semanas de muestreo a través del tiempo (Figura 7).

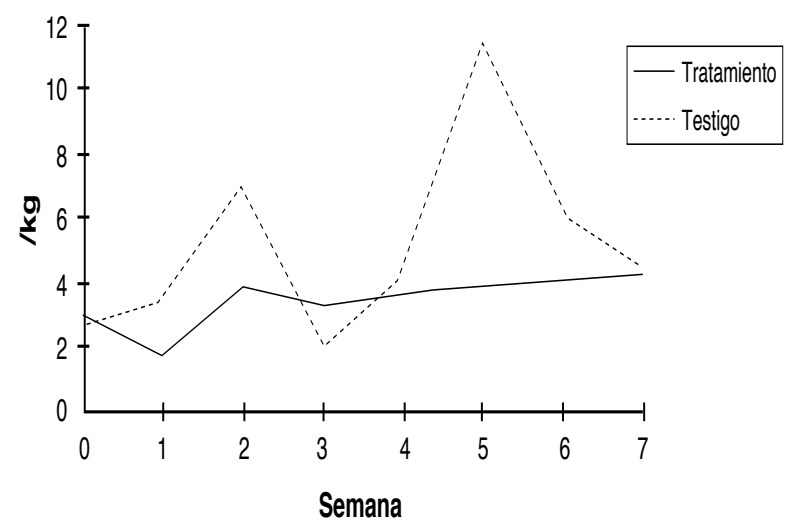

Figura 7. Pérdida de peso en trojas aplicadas con B. bassiana más kaolinita y trojas testigo con maíz fumigado previa la inoculación con $P$. truncatus.

\section{Ensayo III. Trojas inoculadas con Prostephanus trun- catus un mes antes de la aplicación del producto.}

Las diferencias entre tratamientos y semanas de muestreo no fueron significativamente diferentes a través del tiempo para las variables densidad de $P$. truncatus y $S$. zeamais y pérdida de peso $\mathrm{P}(\alpha=0,05)$. No se encontró una interacción significativa entre tratamientos y fecha de muestreo para todas las variables analizadas.

Las poblaciones de $P$. truncatus no difirieron significativamente entre tratamientos y semanas de muestreo a través del tiempo (Figura 8).
Las poblaciones de $S$. zeamais no difirieron significativamente entre los tratamientos y semanas de muestreo a través del tiempo (Figura 9).

En las variables pérdida de peso, $P$. truncatus y $S$. zeamais por kilogramo de grano (insectos día) a través del tiempo no se encontró diferencia estadística significativa entre las trojas con el producto y las trojas testigo $(\alpha=0,05)$.

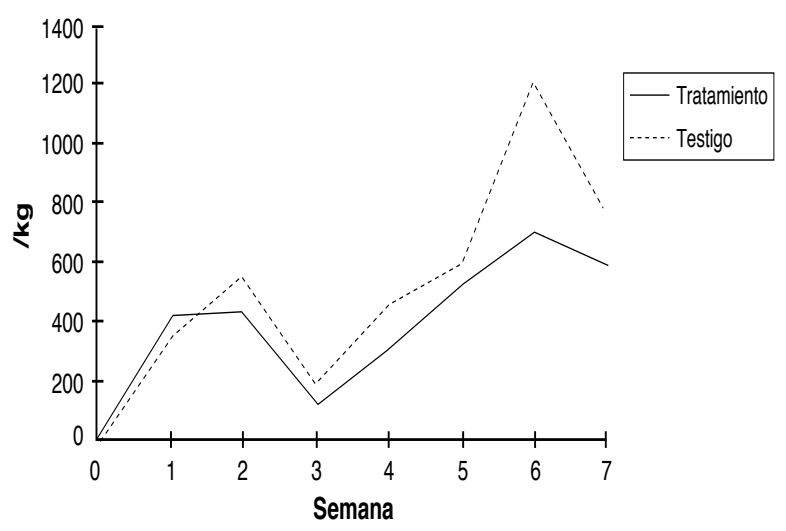

Figura 8. Dinámica poblacional de $P$. truncatus en trojas con $B$. bassiana más kaolinita y trojas testigo en maíz inoculado con $P$. truncatus previa la aplicación del producto.

A través de todas las semanas de muestreo en trojas tratadas con el producto la tasa de mortalidad por B. bassiana fue menor que en el ensayo anterior, con un promedio de $0,31 \%$ para $P$. truncatus y de $0,37 \%$ para $S$. zeamais. Con una muestra viva de P. truncatus en la última semana de muestreo, se encontró un porcentaje de mortalidad de $3,1 \%$ en contraste con el porcentaje de mortalidad de la muestra de insectos muertos (Figura10).

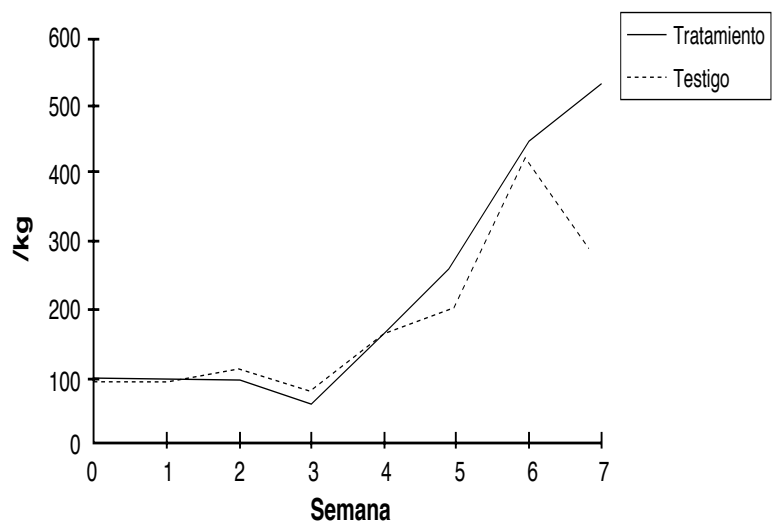

Figura 9. Dinámica poblacional de $S$. zeamais en trojas con $B$. bassiana más kaolinita y trojas testigo en maíz inoculado con $P$. truncatus previa la aplicación del producto. 
La pérdida de peso entre los tratamientos y semanas de muestreo no difirieron significativamente a través del tiempo (Figura 11).

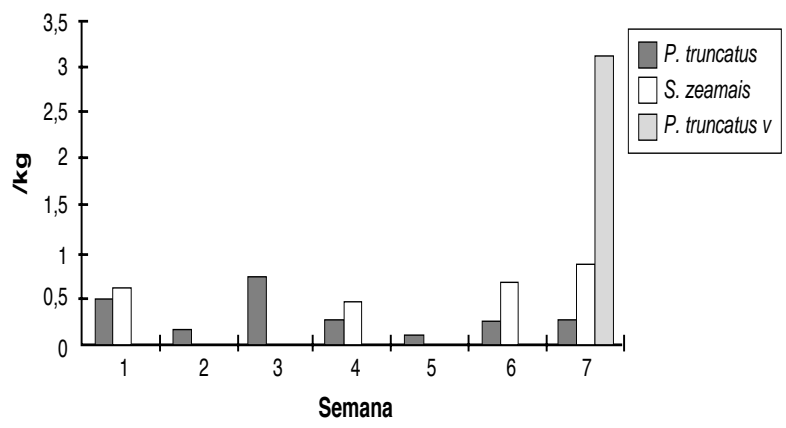

Figura 10. Porcentaje de mortalidad de P. truncatus y $S$. zeamais en trojas con $B$. bassiana más kaolinita en maíz inoculado con $P$. truncatus previa la aplicación del producto. (P. truncatus $\mathrm{v}=$ muestra viva de insectos).

\section{DISCUSIÓN}

Los aislamientos de $B$. bassiana provenientes de plagas del orden Coleóptera (A. grandis, C. sordidus e H. hampeii) obtuvieron porcentajes de control sobre $P$. truncatus ( $84 \%$ en promedio) mayores que el aislamiento de la plaga del orden Lepidóptera (P. xylostella) con $64 \%$. Esto pudo deberse a que los primeros aislamientos eran del mismo orden (Coleóptera) que $P$. truncatus y los aislamientos del orden Coleóptera atacaron los estados maduros de los insectos; mientras que el aislamiento del orden Lepidóptera atacó el estado larval de la plaga.

Los niveles de mortalidad obtenidos en $P$. truncatus son buenos y prometedores ya que resultados similares se han encontrado con plagas como Anthonomus eugenii (picudo del chile) teniendo mortalidades por B. bassiana de $82 \%$, con la misma dosis en los bioensayos.

En las pruebas realizadas en contra de $T$. nigrescens los aislamientos de A. grandis y $C$. sordidus causaron una mortalidad de 62 y $67 \%$, respectivamente (Figura 2); ésto debido a que ambos aislamientos provienen del mismo orden Coleóptera. Podría hacerse uso de este hongo en situaciones en las que el depredador T. nigrescens no pueda ser utilizado como en zonas en que las condiciones ambientales óptimas $\left(26^{\circ} \mathrm{C}\right.$ y $70 \%$ de humedad relativa) no permitan su establecimiento (Rees, 1985), o cuando halla una falta de hospederos alternos. T. nigrescens se puede reproducir en otras plagas de granos almacenados como son
Dinoderus sp., Sitophilus sp., Ryzopertha dominica y Tribolium castaneum (Rees, 1987, 1991; Leliveldt, 1990, citado por García 1996).

La determinación del tiempo letal medio es importante, debido a que con un tiempo menor para el comienzo del control de la plaga se podrían evitar pérdidas que habrían sido causadas por los insectos. Se encontró que el tiempo en que el hongo mató la mitad de la población de adultos de $P$. truncatus (100 hr) con cada uno de los diferentes aislamientos fue similar al encontrado en H. hampeii (Jiménez, 1996).

La mortalidad de $P$. truncatus fue cuatro veces mayor en el ensayo donde se aplicó el producto en forma preventiva, que cuando se aplicó en forma curativa (Figuras 6 y 10). Esto sugiere que al encontrarse $P$. truncatus dentro de las mazorcas con abundancia de alimento, no saldrá para alimentarse y no se pondrá en contacto con los conidios del hongo entomopatógeno.

Las poblaciones de $P$. truncatus en el ensayo donde se aplicó el producto antes de la inoculación de $P$. truncatus fueron menores, debido a que no todos los insectos penetraron las mazorcas ya que la penetración fue de sólo el 2,6\% y 7,9\% en el primero y segundo muestreos respectivamente, lo cual pudo deberse a que la kaolinita actuó como una barrera física, mientras que en el testigo la penetración fue de $35 \%$ y $70 \%$ para el primer y segundo muestreo respectivamente.

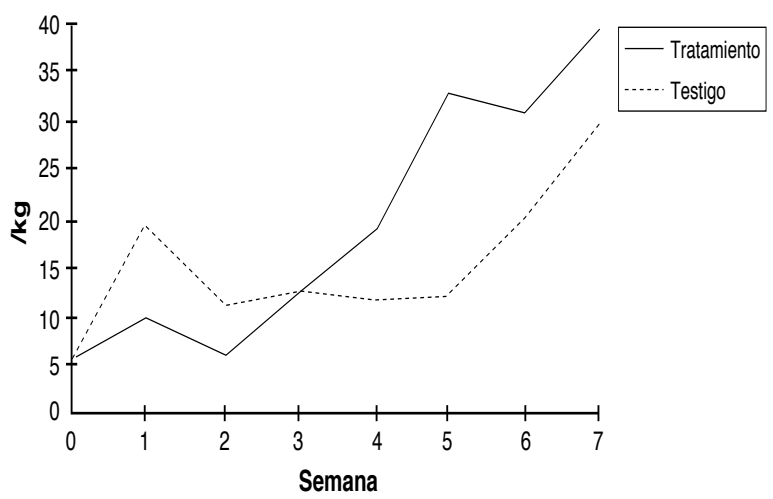

Figura 11. Pérdidas de peso en trojas con B. bassiana más kaolinita y trojas testigo en maíz inoculado con P. truncatus antes de la aplicación del producto.

En las poblaciones de $P$. truncatus no se encontraron diferencias estadísticas significativas a través del tiempo, posiblemente debido a la gran variabilidad entre repeticiones en el Ensayo II, por la metodología que se utilizó para la inoculación de $P$. truncatus, ya que $P$. truncatus mostró una preferencia de moverse hacia el interior de las trojas. 
Las poblaciones de $S$. zeamais fueron similares tanto en el tratamiento con el producto, como en el testigo en ambos ensayos, debido a que esta plaga ya se encontraba en las mazorcas antes de la aplicación del producto, lo cual dificultó el contacto de la plaga con los conidios del hongo entomopatógeno.

La mortalidad de $S$. zeamais fue el doble en el tratamiento en el cual se aplicó el producto al momento de inocularlo con $P$. truncatus que en el tratamiento en el cual se aplicó el producto cuando ya se encontraba inoculado con $P$. truncatus. Esto indica que hubo una transmisión por medio de los cadáveres de $P$. truncatus que contenían el hongo por conidios transportados en la cutícula de $P$. truncatus hasta $S$. zeamais dentro de las mazorcas de maíz. En general, los niveles de mortalidad por $B$. bassiana son mayores en $P$. truncatus que en $S$. zeamais, pero $S$. zeamais mostró aproximadamente un $20 \%$ más de infección por el hongo, en el caso que $P$. truncatus ya se encuentrara en el interior de las mazorcas. Esto indica que $S$. zeamais presentó una mayor tendencia a salir de las mazorcas, desplazado por la alta densidad de $P$. truncatus. Novillo (1991) encontró que altas densidades de $P$. truncatus provocaban la disminución o migración de las poblaciones de $S$. zeamais.

Los porcentajes de mortalidad encontrados, son similares a los que se obtuvieron en pruebas de campo en almacenes, a una dosis parecida de $B$. bassiana realizadas en Africa, siendo estos de 1,2 a 4,0\% (Loreck, 1996). Los bajos porcentajes de mortalidad encontrados en todos los muestreos, se podrían aumentar con una dosis mayor de conidios para lograr mayor contacto con la plaga.

Las pérdidas en el peso de maíz fueron altas, en el período de estudio de siete semanas ya que pérdidas similares se han encontrado en almacenes, pero en un período de almacenamiento de seis meses. Esto se debió a que en el maíz se liberaron una gran cantidad de $P$. truncatus por mazorca (Figuras 7 y 11).

Con los resultados obtenidos en ambos ensayos, se puede concluir que el hongo B. bassiana no se transmite mucho dentro de las mazorcas de maíz. La mejor opción es aumentar la dosis de conidios para controlar la población de $P$. truncatus que penetre en la troja una vez montada. Esto no sucedería en el caso de $S$. zeamais, porque este infecta las mazorcas en el campo, lo cual no permitiría que la plaga entre en contacto con las conidios de B. bassiana.

\section{CONCLUSIONES}

Los aislamientos provenientes del orden Coleóptera fueron los que causaron la mayor mortalidad de Prostephanus truncatus que el aislamiento del orden Lepidóptera.

El mejor aislamiento fue el de A. grandis con una alta mortalidad sobre $P$. truncatus.

En T. nigrescens también se obtuvo una alta mortalidad por el hongo B. bassiana.

Todos los aislamientos actuaron rápidamente sobre P. truncatus.

$P$. truncatus tuvo mayor mortalidad que $S$. zeamais debido a $B$. bassiana.

No hubo un impacto significativo del hongo aplicado en kaolinita entre capas de maíz en trojas sobre las poblaciones de $P$. truncatus y $S$. zeamais, y pérdida de peso.

$S$. zeamais es más propenso a infectarse con el hongo que $P$. truncatus al estar ambos dentro de las mazorcas de maíz.

El hongo puede dispersarse dentro de las mazorcas de maíz por el contacto entre insectos sanos y enfermos, pero esto no produce un impacto significativo en la población.

\section{RECOMENDACIONES}

Evaluar en el campo los aislamientos de B. bassiana provenientes de Hypothenemus hampeii y Cosmopolites sordidus que causaron una mortalidad similar que el aislamiento de Anthonomus grandis.

Utilizar este hongo en situaciones en que no pueda actuar el depredador Teretriosoma nigrescens y probar mayores dosis de este hongo aplicados al maíz en mazorca al momento de ser almacenadas.

Evaluar aplicaciones a las estructuras de almacenamiento para lograr un mayor contacto de los insectos con los conidios del hongo y realizar las aplicaciones al momento de la colocación de las mazorcas en los almacenes.

Evaluar el efecto que tendría este hongo en los diferentes tipos de troja usadas por los agricultores para el almacenamiento de maíz.

Evaluar un testigo con kaolinita para aislar el efecto de esta sobre la penetración de los insectos en las mazorcas. 


\section{LITERATURA CITADA}

CALIX, L. C. 1995. Variación en el potencial biótico de Prostephanus truncatus (Horn) (Coleoptera: Bostrichidae) en Honduras. Tesis de Ing. Agr., Escuela agrícola Panamericana, El Zamorano, Honduras. 59p.

GARCÍA, A. R. 1996. Impacto de prácticas de almacenamiento en la dinámica poblacional y daño ocasionado por Prostephanus truncatus (Horn) (Coleoptera: Bostrichidae) y en Teretriosoma nigrescens (Lewis) (Coleoptera: Histeridae). Tesis de Ing. Agr., Escuela agrícola Panamericana, El Zamorano, Honduras. pp. 3-9.

JIMÉNEZ, C.M. 1996. Evaluación de Beauveria bassiana en el control de plagas de cultivos tropicales. Proyecto, MIP, CATIE, Managua, Nicaragua. p.

KEIL, H. 1988. Losses caused by the larger grain borer in farm stored maize. In Markham, R., Wright, V. and Rios Ibarra, R. (eds.). A selective review of research on $P$. truncatus (Horn) (Coleoptera: Bostrichidae) with annotated updated bibliography. CEIBA 32(1):5.

LORECK, C. 1995. Técnicas de evaluación de hongos entomopatógenos en Prostephanus truncatus por medio de bioensayos. Instituto Internacional de Agricultura Tropical (IITA), Benin. (Comunicación personal).
LORECK, C. 1996. Evaluación de B. bassiana en P. truncatus. Instituto Internacional de Agricultura Tropical (IITA), Benin. (Comunicación personal).

MARKHAM, R.H.; WRIGHT, V.F.; RIOS IBARRA, R.N. 1991. A selective review of research on Prostephanus truncatus (Coleoptera: Bostrichidae) with an annotated and updated bibliography. CEIBA (Hon) 32 (1):1-26.

NOVILLO, P. 1991. Prostephanus trucatus (Horn) (Coleoptera: Bostrichidae) en el ambiente de almacenamiento tradicional de maíz en el Valle del Zamorano, Honduras, C.A. Tesis de Ingeniero agrónomo, Escuela agrícola $\mathrm{Pa}$ namericana, El Zamorano, Honduras. 129 p.

REES, D.P. 1985. Teretriosoma nigrescens Lewis (Coleoptera: Histeridae) a predator of the larger grain borer Prostephanus truncatus (Horn) (Coleoptera: Bostrichidae): Current Status of Knowledge, pp. 103-111. Ed. by R.H. Markham y H.R. Herren. Biological control of the larger grain borer. Proceedings of an IITA/FAO coordination meeting, Cotonou, Republic of Benin 1989. Instituto Internacional de Agricultura Tropical, Ibadan, Nigeria. VIII $177 \mathrm{p}$.

RÍOS, R.M. 1991. Ecología de Prostephanus truncatus (Horn) (Coleoptera: Bostrichidae) en el altiplano de México. Tesis M.Sc., Colegio de Postgraduados, Montecillos, México. 85 p. 